High-performance were defined from the low-performance by improved FAI status or remaining within the upper most two quartiles. Significant variables in univariate analysis were further analyzed using logistic regression to ascertain predictors of improved FAI status.

Results: At 2-year, there were 57(39\%) high and 89(61\%) low performance (FAI: $2.49 \pm 3.39$ vs $-3.62 \pm 4.41, \mathrm{p}<0.01$ ). High-performance maintained outdoor/indoor work $(\mathrm{p}<0.01)$ and indoor leisure $(\mathrm{p}=0.198)$ activities. Highperformance declined in outdoor leisure activities, although of lesser degree than low-performance $(-2.23 \pm 2.24$ vs $-4.53 \pm 2.45, \mathrm{p}<0.01)$. Using logistic regression adjusted for gender, VRFs, SPPB and mood, living alone $[\mathrm{OR}=4.02,95 \%$ CI:1.40-11.50] and younger age [OR=0.937, 95\% CI:0.890.99] were predictors of improved FAI status.

Conclusion: About two-fifths of community-dwelling older adults, typically those younger and living alone, maintained good activity participation at 2 years. While work activities were maintained, outdoor leisure participation notably declined. Further studies are needed to ascertain how living alone promotes maintenance of activity participation.

\section{FUNCTIONAL STATUS OF NON-DEMENTED COMMUNITY-DWELLING OLDER ADULTS WITH PRIOR TRAUMATIC BRAIN INJURY}

R.C. Gardner ${ }^{1,2}$, K.M. Langa ${ }^{3}$, K. Yaffe ${ }^{1,2}, 1$. UCSF, San Francisco, California, 2. San Francisco VA Medical Center, San Francisco, California, 3. University of Michigan, Ann Arbor, Michigan

Traumatic brain injury (TBI) is common and associated with heightened dementia risk, but functional implications of prior TBI among non-demented community-dwelling older adults are unclear. We sought to describe functional status associated with prior TBI in the Health and Retirement Study (HRS) - a population-based cohort study of community-dwelling older adults. We studied 984 older adults without dementia randomly selected from HRS 2014 respondents to complete a TBI survey and who reported no prior TBI $(\mathrm{n}=737)$ or prior symptomatic TBI requiring medical attention $(n=247)$. Mean time since first TBI was $38 \pm 19$ years. Functional impairment was defined as self-reported difficulty on $\geq 1$ of 6 instrumental activities of daily living (IADL): using a map, hot meal preparation, grocery shopping, making phone calls, taking medications, and managing money. Covariates assessed included demographics, medical comorbidities, depression, global cognition, and gait speed. We compared functional impairment across TBI groups using regression models adjusted for covariates that significantly differed between groups. Respondents with TBI were younger, less likely to be female, had higher prevalence of medical comorbidities and depression, but did not differ on global cognition or gait speed compared to respondents without TBI. Respondents with TBI were significantly more likely to report impairment on $\geq 1$ IADL compared to those without TBI (30.0\% vs. $20.4 \%$, adjusted risk ratio [95\% CI]: 1.31 [1.02-1.66]). In this population-based study of community dwelling older adults without dementia, those with prior TBI had $31 \%$ increased risk of IADL impairment even after accounting for higher prevalence of medical comorbidities and depression.

\section{LONGEVITY KLOTHO GENE POLYMORPHISM AND} THE RISK OF DEMENTIA IN OLDER MEN

L. Flicker ${ }^{1}$, B. Morar ${ }^{3}$, G. Hankey ${ }^{3}$, B. Yeap ${ }^{3}$, J. Golledge ${ }^{2}$, A. Jablensky ${ }^{3}$, O. Almeida ${ }^{1}$, 1. Western Australian Centre for Health \& Ageing, University of Western Australia, Perth, Western Australia, Australia, 2. James Cook University, Townsville, Queensland, Australia, 3. University of Western Australia, Perth, Western Australia, Australia

In mice the Klotho gene encodes a membrane protein that seems to suppress certain physiological aspects associated with the ageing phenotype including reduced lifespan and atherosclerosis. In humans Klotho variants (KL-VS) have been associated with increased longevity and better cognitive function. It is unclear whether they modulate dementia risk.

Using an existing cohort of older men, we recruited 527 individuals aged 71-87 years who were free of cognitive impairment. We used data linkage to track the onset of dementia over 10 years. KL-VS genotyping (rs9527025 T/G) followed standard procedures.

In these men, $370(70.2 \%), 145(27.5 \%)$ and $12(2.3 \%)$ had the TT, TG and GG genotypes of KL-VS polymorphism. The Hardy-Weinberg test showed that this allelic distribution was in equilibrium. Overall the age adjusted annual rate of dementia was $17.2 \%$ o $(95 \% \mathrm{CI}=14.0-21.1$; total $=5053$ person-years). The rates were $14.0 \%$ o (95\%CI=10.6-18.4; 3582 person-years), $23.5 \%$ o (95\% CI=16.6-33.2; 1363 personyears) and $46.4 \%$ (95\% CI=19.3-111.5; 108 person-years) for men with the TT, TG and GG genotypes. Compared with the TT genotype, the sub-hazard ratios of dementia associated with the TG and GG genotypes were $1.6(95 \% \mathrm{CI}=1.0$, $2.5 ; \mathrm{p}=0.030)$ and $3.5(95 \% \mathrm{CI}=1.3,9.1 ; \mathrm{p}=0.011)$.

The Klotho KL-VS variant is associated with an increase in the incidence of dementia in older men in a dose-dependent fashion (intermediate for heterozygosis and highest for homozygosis). If these findings can be replicated then the risk of dementia may be able to be modulated by exploiting this metabolic pathway.

\section{ARE ACUTE GERIATRIC UNITS MORE EFFECTIVE FOR HOSPITALIZED SENIORS WITH FRAILTY AND ADVANCED AGE? \\ Y. Ding, 1. Tan Tock Seng Hospital/National Healthcare \\ Group, Singapore, Singapore, 2. Health Services \& \\ Outcomes Research, National Healthcare Group, \\ Singapore, Singapore, 3. Lee Kong Chian School of \\ Medicine, Nanyang Technological University, Singapore, Singapore}

Although acute geriatric units (AGU) are effective in care of hospitalized seniors, it is unclear who among them accrue greater benefit. Therefore, this study seeks to evaluate frailty and advanced age as predictors of benefit from AGU care compared with usual care for short-term outcomes. In a retrospective cohort study, we merged chart and administrative data of seniors aged 65 years and older hospitalized with pneumonia at three acute care hospitals over one year. Frailty is defined as Frailty Index of at least 0.25, while advanced age is 80 years and older. Thirty-day mortality, hospital length of stay (LOS), and episode cost are regressed in turn on AGU care, while controlling for frailty, advanced age, gender, severity of illness, recent hospitalization, and nursing 